\title{
Coronavirus, social distancing, and global geriatric mental health crisis: opportunities for promoting wisdom and resilience amid a pandemic
}

The COVID-19 pandemic has been a health crisis of unparalleled magnitude in recent history. As of September 1, 2020, there were about 25 million people with a confirmed diagnosis of COVID-19, of whom 17 million have recovered, while 850,000 have died. The largest number of cases has been in the USA, followed by Brazil, and then India. Of all the age groups, adults over age 65 are consistently reported to have the highest risk of developing severe illness with complications, requiring hospitalization, needing intensive care unit treatment including ventilator, and dying. This has led to heightened fear and anxiety among older adults, and unfortunately, greater ageism in others by encouraging avoidance of seniors. As if that were not enough, the social distancing requirements to contain the spread of infection are impacting older adults disproportionately more. Many older adults do not have access to the Internet, email, social media, or videoconferencing, which are widely used by younger generations to compensate for the lack of in-person social contacts. Moreover, reliance on technology solutions places a higher burden on older adults with cognitive or sensory impairments. Additionally, most seniors miss aspects of in-person human interactions like a touch and a threedimensional perspective.

This issue of International Psychogeriatrics (IPG) is probably the first issue of any journal that selectively focuses on the impact of COVID-19 and associated social distancing on geriatric mental health across the globe as well as suggested strategies to promote the well-being of older adults, from nearly every continent in the world. The IPG is the flagship journal of the International Psychogeriatric Association (IPA). As Sano et al. (2020) point out in their Guest Editorial, the IPA was one of the key international entities that, under the auspices of the WHO, led to the conceptualization of the principles of psychiatric care for older adults. Through efforts including the current issue of the IPG, the IPA will strive to promote the well-being of older adults affected by the COVID-19 directly or indirectly across all the countries. Another unique feature of this issue is that it includes a statement paper from the American Association for Geriatric Psychiatry (Parker, et al., this issue), which is being published simultaneously in IPG and in the American fournal of Geriatric Psychiatry - a first for both the journals.

The various Commentaries in this issue, both solicited and unsolicited, have come from Africa (Ghana, Nigeria), Asia (China, India, Japan, the Philippines, and Taiwan), Australia, Eurasia (Turkey), Europe (Germany, Ireland, Italy, the Netherlands, Norway, Portugal, Spain, Sweden, and the UK), the Mideast (Israel, Lebanon), North America (Canada, USA.), and South America (Brazil, Dominican Republic). Some of the Commentaries are not region-based but focus on specific aspects of psychogeriatrics related to COVID-19 and social distancing. Thus, Brennan et al. discuss the social work perspective, Ayalon addresses ageism, and Peisah et al. consider the human rights angle. Wand et al. describe the implications for suicidal behavior, while Moore and colleagues make recommendations for supporting families in end-of-life care and bereavement. Suzuki et al. discuss the behavioral pattern of patients with frontotemporal dementia, whereas Ayalon and colleagues focus on long-term care settings. Hwang and colleagues elaborate on the topic of loneliness and social isolation, and Vernooij-Dassen and colleagues point out the risks of social distancing in older adults, with a call to balance.

Most papers describe the horrendous effects of COVID-19 and the associated social isolation and loneliness in older adults in various parts of the world. Yet, there are also inspiring examples of professional creativity developed and implemented by geriatric psychiatrists and other mental health clinicians in different countries. In China, a collaboration initiated by the Chinese Society of Geriatric Psychiatry involved the Chinese Society of Psychiatry, the Chinese Psychiatric Association, and the Chinese Association for Mental Health, and helped facilitate a coordinated national response to improve mental health care of older adults and caregivers during the COVID-19 outbreak (Wang et al., 2020). Similarly, in Taiwan, the Taiwanese Society of Geriatric Psychiatry and Taiwan Alzheimer's 
Disease Association worked with national and local government agencies to provide necessary information to older adults and their families to aid in controlling the spread of the virus (Hwang et al., 2020). In the USA, the COVID-19 pandemic has catalyzed the adoption of telepsychiatry at record speed (Parker et al., 2020). Importantly, telepsychiatry is now, for the first time, being reimbursed by the government as well as private insurance plans as physical distancing is becoming the new norm.

It is important to stress that loneliness is not a new phenomenon generated by the coronavirus or by the mandated social distancing. During the past couple of decades, there has been an ongoing behavioral pandemic of suicides and opioid-related deaths across the lifespan, reflecting growing levels of loneliness in the society (Jeste et al., 2020). The good news is that loneliness is potentially modifiable. A number of interventions to prevent or reduce loneliness have been tried with varying levels of success. Our recent studies including several thousand people across the adult lifespan have found a consistently strong inverse correlation between wisdom and loneliness (Lee et al., 2019; Nguyen et al., 2020; Jeste et al., 2020). Wisdom is a complex personality trait comprised of specific components - prosocial behaviors like empathy and compassion, emotional regulation, self-reflection, decisiveness while accepting uncertainty and diversity of perspectives, and spirituality. Of these components, the one with the strongest correlation has been compassion. Wisdom, including prosocial behaviors, is potentially modifiable and can be enhanced with psychosocial interventions in many people (Treichler et al., 2020; Lee et al., 2020).

All crises harvest new opportunities too. The increasing attention being paid to mental health in general and to social isolation in particular, as a result of the COVID-19 pandemic, is leading to the consideration of developing suitable technologies for seniors. Despite the downsides of employing technology for older adults mentioned earlier, there are aging baby boomers who are open to learning new technology. In many retirement communities, there has been a rapid growth in the use of smartphones (including FaceTime), emailing of pictures and videos, and celebrations of family or group events on Zoom by older adults who had stayed away from those technologies in the past. As the lockdowns continue or return, people are realizing that, while physical distancing is essential, social distancing is damaging. One can socialize remotely, and while the remote socialization is not qualitatively the same as physical proximity or contact, it may be the best alternative at the present time for many people. There are also reports of resilient older adults who are becoming role models for stressedout youth.

As the research on loneliness and social isolation moves into the next phase, several new questions will come up. What key biological, psychosocial, and environmental factors are driving the loneliness pandemic, compounded by social isolation during the COVID-19 pandemic? Are there sex-specific differences or other individual characteristics or group-level explanatory factors? What would be the broader public health implications of a widespread social recession in the wake of the COVID-19 pandemic? What are the major challenges to developing neurobiologically and psychosocially grounded interventions for loneliness? Using the precision medicine approach, how can interventions be tailored for different subgroups? How can large population-based datasets and remote interventions be leveraged to reduce social isolation at the community level? How can societal changes associated with physical distancing (living, working, and public spaces) be counteracted to mitigate or prevent loneliness?

I hope that future issues of this journal will feature new and exciting research addressing these and other important questions seeking to end the vicious grip of loneliness and stress so as to transform the global society into one with happier, healthier, and wiser members of all ages.

\section{Conflict of interest}

The author declares no potential conflicts of interest with respect to the research, authorship, and/or publication of this report.

\section{Acknowledgments}

This work was supported, in part, by the Center for Healthy Aging and the Sam and Rose Stein Institute for Research on Aging at the University of California San Diego.

\section{Dilip V. Jeste}

Department of Psychiatry, University of California San Diego, San Diego, USA

Sam and Rose Stein Institute for Research on Aging, University of California San Diego, San Diego, USA Department of Neurosciences, University of California San Diego, San Diego, USA

Email: djeste@health.ucsd.edu 


\section{References}

Holt-Lunstad, J. (2017). The potential public health relevance of social isolation and loneliness: Prevalence, epidemiology, and risk factors. Public Policy \& Aging Report, 27, 127-130.

Jeste, D. V. et al. (2020). Study of loneliness and wisdom in 482 middle-aged and oldest-old adults: a comparison between people in Cilento, Italy and San Diego, USA. Aging and Mental Health (in press).

Jeste, D. V., Graham, S. A., Nguyen, T. T., Depp, C. A., Lee, E. E. and Kim, H. C. (2020). Beyond artificial intelligence: exploring artificial wisdom. International Psychogeriatrics. https://doi.org/10.1017 /S1041610220000927

Jeste, D. V., Lee, E. E., and Cacioppo, S. (2020). Battling the modern behavioral epidemic of loneliness: suggestions for research and interventions. $\mathcal{F} A M A$ Psychiatry. https:// doi.org/10.1001/jamapsychiatry.2020.0027

Jeste, D. V., Lee, E. E., Palmer, B. W. and Treichler, E. B. H. (2020). Moving from humanities to sciences: a new model of wisdom fortified by sciences of neurobiology, medicine, and evolution. Psychological Inquiry, 31, 134-143. https://doi.org/10.1080/1047840x.2020.1757984

Lee, E. E. et al. (2020). Outcomes of randomized clinical trials to enhance social, emotional, and spiritual components of wisdom: a systematic review and metaanalysis. FAMA Psychiatry. https://doi.org/10.1001/ jamapsychiatry.2020.0821

Lee, E. E. et al. (2019). High prevalence and adverse health effects of loneliness in community-dwelling adults across the lifespan: role of wisdom as a protective factor. International
Psychogeriatrics, 31, 1447-1462. https://doi.org/10.1017 /S1041610218002120

Morlett Paredes, A. et al. (2020). Qualitative study of loneliness in a senior housing community: the importance of wisdom and other coping strategies. Aging and Mental Health. https://doi.org/10.1080/13607863.2019.1699022

National Academies of Sciences, E., \& Medicine. (2020). Social Isolation and Loneliness in Older Adults: Opportunities for the Health Care System. Washington, DC: The National Academies Press.

Nguyen, T. et al. (2020). Predictors of loneliness by age decade: study of psychological and environmental factors in 2,843 community-dwelling Americans aged 20-69 years. Fournal of Clinical Psychiatry (in press).

Parker, C., Forbes, M., Vahia, I., Forester, B., Jeste, D. V. and Reynolds, C. (2020). Facing the change together: reflections of coping and resilience from american geriatric psychiatrists during COVID-19. American fournal of Geriatric Psychiatry and International Psychogeriatrics (this issue).

Sano, M. et al. (2020). Psychogeriatricszs in a world with Covid-19. International Psychogeriatrics (this issue).

Treichler, E. B. H. et al. (2020). A pragmatic trial of a group intervention in senior housing communities to increase resilience. International Psychogeriatrics, 32, 173-182 . https://doi.org/10.1017/S1041610 219002096

Wang, H. et al. (2020). Coronavirus epidemic and geriatric mental healthcare in china: how a coordinated response by professional organizations helped older adults during an unprecedented crisis. International Psychogeriatrics (this issue). 